\title{
Accidental Placement of Nasogastric Tube into the Brain: Case Report and Review of the Literature
}

\author{
Khursheed Ansari ${ }^{1} \quad$ Rafique Umer Harvitkar ${ }^{2}$ Saiprasad Shetty ${ }^{2}$ Tushar P. Raut ${ }^{3} \quad$ Shabnam Qureshi ${ }^{4}$
}

${ }^{1}$ Department of Neurosurgery, Dr. L.H. Hiranandani Hospital, Mumbai, Maharashtra, India

2Department of General and Laparoscopic Surgery, Dr. L.H. Hiranandani Hospital, Mumbai, Maharashtra, India

${ }^{3}$ Department of Neurology, Kokilaben Dhirubhai Ambani Hospital, Mumbai, Maharashtra, India

${ }^{4}$ Department of Obstetrics and Gynaecology, AllCure Hospital, Mumbai, Maharashtra, India

Int J Recent Surg Med Sci:2020;6:74-76

\begin{abstract}
Address for correspondence Rafique Umer Harvitkar, MBBS, MS, MRCS, Department of General and Laparoscopic Surgery, Dr. L.H. Hiranandani Hospital, Powai, Mumbai 400076, Maharashtra, India (e-mail: dr_rafique639@yahoo.com).
\end{abstract}

\begin{abstract}
Keywords

- NGT insertion

- NGT complication

- pneumocephalus

- C-arm guidance

- fractured cribriform plate
\end{abstract}

The nasogastric tube (NGT) plays a very vital role in the early resuscitation of head injury patients for various reasons. Though considered a simple procedure, it can be associated with multiple dreadful complications. Our patient was a 44-year-old male, referred from a peripheral health center for further management of severe head and faciomaxillary injuries following a motor vehicular accident. On arrival at the hospital, the endotracheal tube and NGT were in situ. A computed tomography scan of the brain revealed the NGT piercing through the cribriform plate of ethmoid and coiling into the cranial cavity with pneumocephalus. The NGT was retrieved under C-arm guidance and his injuries were managed respectively. The patient was discharged after prolong hospitalization with an acceptable outcome. With this case report, we want to remind emergency health care providers to avoid this catastrophic complication of NGT insertion in a faciomaxillary trauma patient by doing it transorally. We report a novel method of safely removing NGT which has not been reported in the past.

\section{Introduction}

"Time is brain" for a head injury patient. They require clinical assessment, diagnostic imaging, and primary treatment without any delay in the emergency department.

The nasogastric tube (NGT) insertion is considered one of the most commonly performed bedside or emergency department procedures. Despite being a routine innocuous procedure, it has been known to cause dreadful complications such as esophageal or gastric perforation, laryngeal injuries, pneumothorax, or accidental intracranial insertion. ${ }^{1,2}$ Inadvertent insertion of NGT into the cranial cavity was first described by Martinelle et al in $1974^{3}$ followed by Seebacher et al in late $1980 .{ }^{4}$ Since then, few cases have been reported in medical journals.

published online

September 22, 2020
Dol https://doi.org/

10.1055/s-0040-1716802 ISSN 2455-7420.
We report a rare case of misplacement of NGT into the cranial cavity in a faciomaxillary injured patient. The patient was referred from peripheral hospital following a road traffic accident (RTA) with an endotracheal tube and NGT in situ ( -Fig. 1A). This case aims to alert medical personal about NGT complications and measures to avoid these complications. Authors have also tried to review the existing literature and throw light on the methods to remove the tube. This article proposes a novel method of retrieval of the NGT from the brain.

\section{Case Report}

A 44-year-old male referred from a peripheral hospital with a history of a motor vehicular accident. The patient had

(C) 2020. Medical and Surgical Update Society.

This is an open access article published by Thieme under the terms of the Creative Commons Attribution-NonDerivative-NonCommercial-License, permitting copying and reproduction so long as the original work is given appropriate credit. Contents may not be used for commercial purposes, or adapted, remixed, transformed or built upon. (https://creativecommons.org/licenses/by-nc-nd/4.0/)

Thieme Medical and Scientific Publishers Pvt. Ltd., A-12, 2nd Floor, Sector 2, Noida-201301 UP, India 

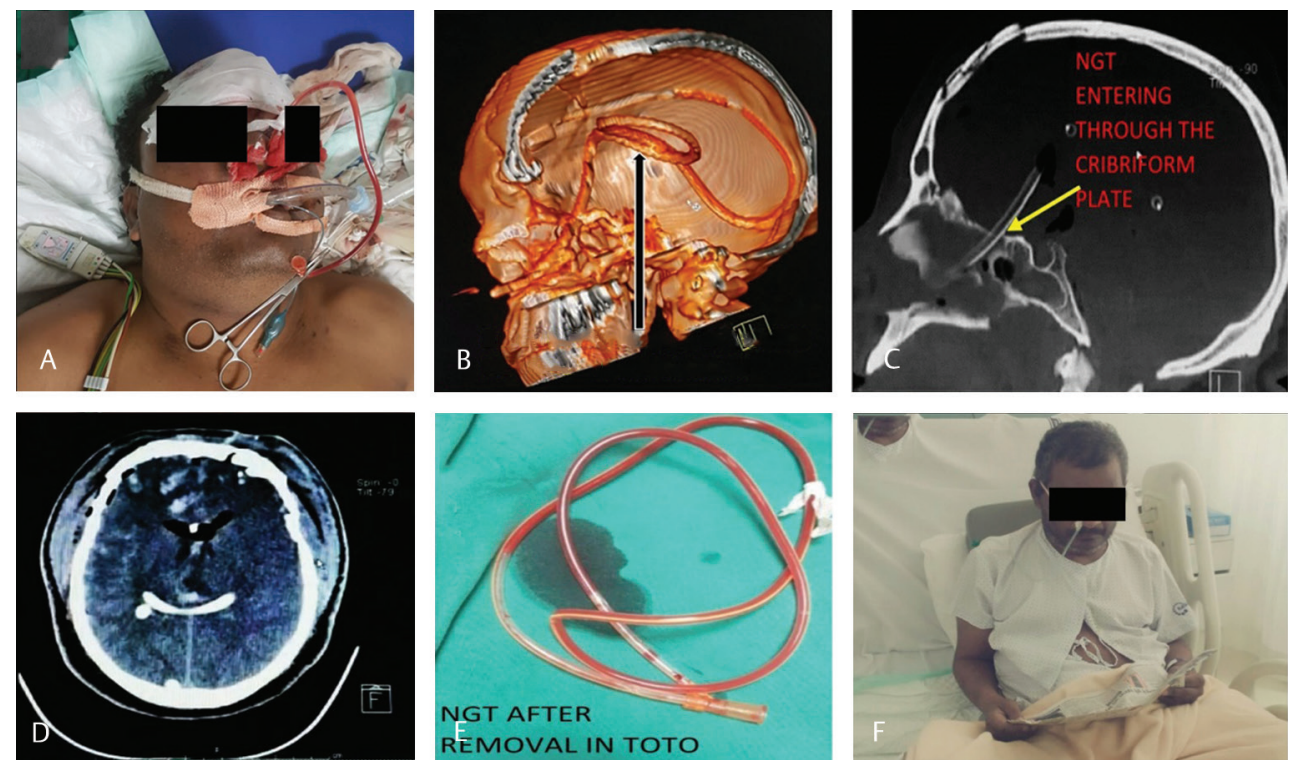

Fig. 1 (A-F) Sequence of events. (A) The patient received in the emergency department with an endotracheal and nasogastric tube (NGT) in situ. (B) Three-dimensional image showing entire NGT into cranial vault. (C) NGT passing through a fracture cribriform plate. (D) NGT in the axial section crossing mid line. (E) Retrieved NGT. (F) Patient post-recovery reading newspaper.

suffered severe faciomaxillary injuries and he was brought with an endotracheal tube and NGT in situ $(-$ Fig. 1A). The patient was in tachycardia and hypotension due to significant blood loss from the injuries. On examination, he had large forehead laceration and multiple bruises over the temporoparietal region. He had a nose bleed with cerebrospinal fluid (CSF) rhinorrhea and oral bleed. NGT aspiration was showing serosanguinous fluid. His Glasgow coma score (GCS) was E1M2Vt, and pupils could not be examined due to the bilateral "raccoon eye." An 18 gauge intravenous access was secured and the colloid infusions were given to stabilize hemodynamic instability. Lacerations were sutured to control the bleeding. A secondary survey completed and after ruling out other injuries including blunt chest and abdominal trauma, the patient was immediately planned for a computed tomography (CT) of the brain, face, and cervical spine. CT scan imaging showed NGT shoving through the fractured cribriform plate of ethmoid and coiled into the bilateral cerebral hemisphere ( $\boldsymbol{- F i g}$. 1B-D). There were associated pneumocephalus and multiple comminuted fractures of the skull. There were multiple fractures of faciomaxillary bones.

The patient was shifted to the operating room, and under C-arm fluoroscopy the NGT tube was removed gently observing that the tube describing its convolution curves while coming out ( - Fig. 1E). There was no resistance encountered while pulling out the tube, and the entire feeding tube could be extracted uneventfully. Another NGT passed transorally under laryngoscopic guidance by the anesthetist. He underwent tracheostomy due to severe faciomaxillary injuries and anticipating prolonged ventilation requirements due to poor GCS. The postprocedure CT scan brain did not show any fresh bleed or contusions. His CSF rhinorrhea was resolved spontaneously over a few days and did not require any surgical intervention during recovery ( - Fig. 1F). His final modified Rankin scale was 2 .

\section{Discussion}

NGT insertion is a routinely performed procedure in an emergency department on unresponsive or noncooperative patients. The NGT is usually inserted in head injury patients to avoid gastric dilatation, prevent gastric content aspiration, determine the nature of the gastric contents and early access for enteral feeding. ${ }^{1}$ Despite how commonly the NGTs are used, they are associated with dreadful and severe complications. Accidental insertion of NGT into the trachea and distal airways is said to occur in 0.3 to $15 \%$ of insertions. ${ }^{5,6}$ However, the accidental insertion of NGT into the cranial cavity is a rare complication. ${ }^{2.5}$ To the best of our knowledge, only 40 cases of accidental insertion of NGT into the brain have been reported in the literature to date.

The inadvertent placement of NGT into the cranial cavity has been described due to multiple factors as:

1. The base of the skull or cribriform plate fracture.

2. A thin cribriform plate due to chronic sinusitis may precipitate an intracranial insertion.

3. Cranial defects of the skull base may communicate between the nasal and cranial cavity.

Approximately $25 \%$ of patients with fractures of the medial third of the frontal bone have a cribriform plate fracture.7,8 In most of the instances, the tube gets entrapped into the vault of the skull rather than penetrating through the parenchyma of the brain. ${ }^{9}$ The NGT into the cranial cavity maybe associated with severe complications such as meningitis, hematoma in the brain, sensory motor deficit, hemiparesis, blindness, or death in $64 \%$ of the cases. ${ }^{1}$

One should have a high index of suspicion while inserting the NGT in RTA patients. Physicians should be vigilant about "red flag signs" of skull base fractures such as Raccoon eyes, Battle sign, CSF rhinorrhea, or obvious maxillofacial trauma. 
As per the Advance Trauma Life Support guidelines, NGT should be avoided in the patient with skull base fracture. However, if necessary, placement should be done through oropharynx as a routine protocol. In these instances, the NGT should be guided into the stomach under direct vision or fluoroscopic or endoscopic guidance. ${ }^{10}$

Radiological imaging should always be performed to determine the position of the tube. However, when the radiological imaging confirms the tube into the cranial cavity, prompt attempts should be made to remove the tube. An emergency CT of the brain with broad-spectrum antibiotics is necessary for all the patients., ${ }^{5,11}$ Optimal management of intracranially placed NGT is controversial and debated. However, some authors recommended retrieval through the nose while others believed segmented removal through a craniotomy. Ferreras et al state that there is no scientific evidence available to suggest that either technique offers any prognostic advantage. ${ }^{5}$ In this case, NGT was removed through the nose under fluoroscopic guidance to ensure NGT is not getting stuck and describing its curves while coming out so that secondary injury to the brain is prevented by blindly pulling the same. This has not been reported in the past. This method is vital in preventing secondary injury to the brain during retrieval of the NGT. This was probably one of the reasons for a favorable outcome in our patient. Nevertheless, further study is needed to prove the efficacy of the method. The main aim should be to prevent further complications irrespective of the method adopted for retrieval.

\section{Conclusion}

Although the insertion of NGT is a simple procedure with a low complication rate, the physician or paramedic should always confirm that there is no associated faciomaxillary injury. Clinical signs should never be missed. NGT should be avoided in suspected skull base fractures. The oropharyngeal route should be adopted in unavoidable circumstances under direct vision or fluoroscopic or endoscopic guidance. Retrieval of NGT should also be done under fluoroscopy guidance in case of inadvertent placement of the tube in the cranial cavity. The NGT position should always be confirmed with radiological imaging before starting any feeds. We were able to salvage the patient with prompt and timely measures. The patient could be discharged with a favorable outcome at the end of hospitalization.

\section{Note}

Written informed consent was obtained from the patient for publication of this case and any accompanying images.

\section{Funding}

None.

\section{Conflict of Interest}

None declared.

\section{References}

1 Genú PR, de Oliveira DM, Vasconcellos RJH, Nogueira RVB, Vasconcelos BCE. Inadvertent intracranial placement of a nasogastric tube in a patient with severe craniofacial trauma: a case report. J Oral Maxillofac Surg 2004;62(11):1435-1438

2 Rahimi-Movaghar V, Boroojeny SB, Moghtaderi A, Keshmirian B. Intracranial placement of a nasogastric tube. A lesson to be re-learnt? Acta Neurochir (Wien) 2005;147(5):573-574, discussion 574

3 Martinelle F, Montaut J, Hazeaux C, et al. Pénétration intracranienne D'une sonde gastrique à travers une déhiscence traumatique de la lame Criblée. Cah Anesthesiol 1974;22:345

4 Seebacher J, Nozik D, Mathieu A. Inadvertent intracranial introduction of a nasogastric tube, a complication of severe maxillofacial trauma. Anesthesiology 1975;42(1):100-102

5 Ferreras J, Junquera LM, García-Consuegra L. Intracranial placement of a nasogastric tube after severe craniofacial trauma. Oral Surg Oral Med Oral Pathol Oral Radiol Endod 2000;90(5):564-566

6 Sliwa JA, Marciniak C. A complication of nasogastric tube removal. Arch Phys Med Rehabil 1989;70(9):702-704

7 McWey RE, Curry NS, Schabel SI, Reines HD. Complications of nasoenteric feeding tubes. Am J Surg 1988;155(2):253-257

8 Arslantas A, Durmaz R, Cosan E, Tel E. Inadvertent insertion of a nasogastric tube in a patient with head trauma. Childs Nerv Syst 2001;17(1-2):112-114

9 Marlow TJ, Goltra DD Jr, Schabel SI. Intracranial placement of a nasotracheal tube after facial fracture: a rare complication. J Emerg Med 1997;15(2):187-191

10 Gustavsson S, Albért J, Forsberg H, Ryrberg CH. The accidental introduction of the nasogastric tube into the brain. Case report. Acta Chir Scand 1978;144(1):55-56

11 Thomas B, Cummin D, Falcone RE. Accidental pneumothorax from a nasogastric tube. N Engl J Med 1996;335(17):1325-1326 